\title{
Temperatura e umidade do solo no consórcio de milho com Crotalaria spectabilis e
}

\section{Cajanus cajan em sistema orgânico}

Soil temperature and moisture in the intercropping of maize with Crotalaria spectabilis and Cajanus cajan in an organic system

\section{Temperatura y humedad del suelo en el cultivo intercalado de maíz con Crotalaria spectabilis y \\ Cajanus cajan en sistema orgánico}

\section{Resumo}

A espécie e densidade de adubos verdes no consórcio com o milho modificam os padrões de competição interespecífica podendo alterar as respostas de temperatura e umidade do solo. Objetivou-se, neste estudo, analisar os efeitos da Crotalaria spectabilis e do Cajanus cajan na temperatura e umidade do solo, ao longo dos estádios de desenvolvimento do milho cultivado em consórcio, em sistema orgânico. O delineamento experimental foi blocos casualizados, com quatro repetições. Os tratamentos consistiram em três consórcios de milho: MC - Crotalaria spectabilis $\left(5\right.$ plantas $\left.\mathrm{m}^{-1}\right)$; MG - Cajanus cajan $\left(5\right.$ plantas $\left.\mathrm{m}^{-1}\right)$; MCG - Crotalaria spectabilis e Cajanus cajan (10 plantas $\mathrm{m}^{-1}$, cinco plantas de cada adubo verde), semeados na linha do milho e três tratamentos controles: M - milho; C - Crotalaria spectabilis e G - Cajanus cajan, solteiros. As leituras das temperaturas do solo foram realizadas na linha e entrelinha de semeadura, e, da umidade do solo, na linha de semeadura do milho, nos horários de 8, 12 e 17 horas, nos estádios fenológicos V5, V8, VT, R2, R4 e R6 do milho. As profundidades de avaliação foram 0-0,10, 0,10-0,20 e 0,20-0,40 m. Os horários, as profundidades e as posições linha-entrelinha influenciaram a temperatura do solo, enquanto as profundidades e os estádios fenológicos influenciaram a capacidade de retenção de umidade no solo. Os consórcios reduziram a temperatura do solo, principalmente o MG, que também reduziu a umidade do solo entre os estádios V8 e R2 do milho.

Palavras-chave: Agricultura orgânica; Amplitude térmica; Consorciação de culturas; Fabáceas.

\begin{abstract}
Species and density of green manures intercropped with maize modify the patterns of interspecific competition, which may alter the responses of temperature and soil moisture. This study analyzed the effects of Crotalaria spectabilis and Cajanus cajan on soil temperature and moisture along the development stages of maize grown in intercropping in an organic system. The experimental design was randomized blocks with four replications. The treatments consisted of three maize intercropping: MC - Crotalaria spectabilis ( 5 plants $\left.\mathrm{m}^{-1}\right)$; MG - Cajanus cajan $\left(5\right.$ plants $\left.\mathrm{m}^{-1}\right)$; MCG Crotalaria spectabilis and Cajanus cajan (10 plants $\mathrm{m}^{-1}$, five plants of each green manure), sown in the maize row and three control treatments: M - maize; C - Crotalaria spectabilis and G - Cajanus cajan, single. Soil temperature readings were taken in the row and between the sowing rows, and soil moisture readings in the maize sowing row at 8 , 12 and 17 hours, in V5, V8, VT, R2, R4 and R6 phenological stages. The evaluation depths were 0-0.10, 0.10-0.20
\end{abstract}


and 0.20-0.40 m. The times, the depths and the line-between-row positions influenced the soil temperature, while the depths and the phenological stages influenced the soil moisture retention capacity. Intercropping reduced soil temperature, especially MG, which also reduced soil moisture between V8 and R2 maize stages.

Keywords: Organic agriculture; Thermal amplitude; Intercropping of cultures; Fabaceae.

\section{Resumen}

Las especies y la densidad de los abonos verdes intercalados con el maíz modifican los patrones de competencia interespecífica, lo que puede alterar las respuestas de la temperatura y la humedad del suelo. El objetivo de este estudio fue analizar los efectos de Crotalaria spectabilis y Cajanus cajan sobre la temperatura y la humedad del suelo, a lo largo de las etapas de desarrollo del maíz cultivado en cultivos intercalados, en un sistema orgánico. El diseño experimental fue de bloques al azar con cuatro repeticiones. Los tratamientos consistieron en tres cultivos intercalados de maíz: MC - Crotalaria spectabilis (5 plantas m -1); MG - Cajanus cajan (5 plantas m -1); MCG Crotalaria spectabilis y Cajanus cajan (10 plantas $\mathrm{m}$-1, cinco plantas de cada abono verde), sembradas en la hilera de maíz y tres tratamientos control: M - maíz; C - Crotalaria spectabilis y G - Cajanus cajan, solteros. Se realizaron lecturas de temperatura del suelo en la hilera y entre las hileras de siembra, y lecturas de humedad del suelo en la hilera de siembra de maíz, a las 8, 12 y 17 horas, en las etapas fenológicas V5, V8, VT, R2, R4 y R6 del maíz. Las profundidades de evaluación fueron 0-0,10, 0,10-0,20 y 0,20-0,40 m. Los tiempos, las profundidades y las posiciones de la línea entre hileras influyeron en la temperatura del suelo, mientras que las profundidades y las etapas fenológicas influyeron en la capacidad de retención de humedad del suelo. El cultivo intercalado redujo la temperatura del suelo, especialmente MG, lo que también redujo la humedad del suelo entre las etapas de maíz V8 y R2.

Palavras clave: Agricultura orgánica; Gama térmica; Cultivo intercalado; Fabaceae.

\section{Introdução}

A consorciação envolve o cultivo simultâneo de duas ou mais espécies, durante parte considerável do ciclo produtivo, numa mesma área (Kappes; Zancanaro, 2016). Essa prática tem sido difundida como alternativa para a produção de palha para o sistema de semeadura direta e acúmulo de matéria orgânica no solo (Chioderoli et al., 2012), principalmente no domínio Cerrado, onde ocorre dificuldade para produção de palhada, no outono/inverno, em função do déficit hídrico (Pacheco et al., 2008).

O consórcio do milho com fabáceas (leguminosas) beneficia os atributos físicos do solo (Abdollahi et al., 2014) e cumpre a função de cobertura (Nunes et al., 2018), reduzindo as amplitudes térmicas do solo. Ainda, favorece a manutenção e melhoria dos atributos químicos (Heinrichs et al, 2005; Büchi et al., 2018) e biológicos do solo (Santos et al., 2008), sem que a produtividade de grãos seja prejudicada (Snapp; Surapur, 2018). Adequa-se, também, às premissas da produção orgânica, a qual preconiza a conservação da água, solo, ar e o incremento da biodiversidade vegetal (Brasil, 2003). A Crotalaria spectabilis Roth. e o guandu anão (Cajanus cajan (Millsp.) L.) sobressaem-se entre as fabáceas utilizadas no consórcio com o milho (Gallo et al., 2017; Kappes; Zancanaro, 2016).

Dentre os atributos físicos-hídricos do solo, a temperatura e a umidade destacam-se, pois influenciam diretamente o desenvolvimento do milho (Oliveira et al., 2005; Heinrichs et al, 2005). A temperatura do solo está relacionada com os processos de interação solo-planta (Gasparim et al., 2005; Lal, 1974), uma vez que temperaturas do solo superiores a $42^{\circ} \mathrm{C}$ podem afetar negativamente as plântulas e raízes (Kaspart; Bland, 1992), promover alterações no metabolismo da biota do solo (Heinze et al., 2017; Zhou et al., 2013), na mineralização da matéria orgânica (Conant et al., 2011) e na evaporação da água. Já o déficit hídrico, quando se estabelece na polinização e desenvolvimento inicial do grão, reduz a produtividade do milho (Wagner et al., 2013).

As espécies de adubos verdes alteram a cobertura do solo, bem como o volume explorado pelas raízes, podendo modificar a umidade e a temperatura do solo. A raiz pivotante do guandu anão atinge profundidades de solo superior a $0,30 \mathrm{~m}$, já as crotalárias são mais sensíveis a compactação do solo e concentram suas raízes na superfície (Reinert et al., 2008). O milho, concentra aproximadamente $60 \%$ da densidade das raízes na profundidade 0-0,30 m de solo (Bassoi, et al., 1994).

O guandu anão e a Crotalaria spectabilis apresentam crescimento inicial lento, com baixa cobertura do solo nos primeiros estádios de desenvolvimento (Alvarenga et al., 1995; Gitti et al., 2012). Em contrapartida, a maior taxa de acúmulo 
de matéria seca do milho ocorre nos estádios iniciais do desenvolvimento (Alvarenga et al., 2006). Dessa forma, a semeadura simultânea dos adubos verdes na linha de plantio do milho pode alterar os padrões de competição, depreciando ou otimizando o desenvolvimento do cereal.

Nesse contexto, objetivou-se analisar os efeitos da Crotalaria spectabilis e do Cajanus cajan na temperatura e umidade do solo, ao longo dos estádios de desenvolvimento do milho cultivado em consórcio, em sistema orgânico.

\section{Metodologia}

\subsection{Local do estudo}

O experimento foi conduzido na safra 2017/2018, no município de Araras-SP (latitude 22²1'25" sul, longitude 47 23'03" oeste, altitude de 646 metros) em um Nitossolo Vermelho Distroférrico latossólico (NVdf), de textura argilosa (Yoshida; Stolf, 2016), cujas características físicas estão descritas na Tabela 1.

As químicas na profundidade de 0,0-0,10 m foram: $\mathrm{pH}\left(\mathrm{em} \mathrm{CaCl}_{2}\right)=5,4 ; \mathrm{P}$ resina $=39 \mathrm{mg} \mathrm{dm}^{-3}$; matéria orgânica $=37 \mathrm{mg}$ $\mathrm{dm}^{-3} ; \mathrm{K}=4,3 \mathrm{mmol}_{\mathrm{c}} \mathrm{dm}^{-3} ; \mathrm{Ca}=34,0 \mathrm{mmol}_{\mathrm{c}} \mathrm{dm}^{-3} ; \mathrm{Mg}=11,0 \mathrm{mmol}_{\mathrm{c}} \mathrm{dm}^{-3} ; \mathrm{S}=9,0 \mathrm{mg} \mathrm{dm}^{-3} ; \mathrm{H}+\mathrm{Al}=16,0 \mathrm{mmol}_{\mathrm{c}} \mathrm{dm}^{-3} ;$ soma de bases (S.B.) $=49,3$ mmol $_{\mathrm{c}} \mathrm{dm}^{-3} \mathrm{e} \mathrm{V}$ (saturação de bases) $=75,0 \%$.

Tabela 1. Teores de argila, silte, areia, classificação textural e características físicas do solo nas profundidades $0-0,10,0,10$ 0,20 e 0,20-0,40 m, na área do experimento. Araras-SP, safra 2017/18.

\begin{tabular}{|c|c|c|c|c|c|c|c|c|c|}
\hline \multicolumn{5}{|c|}{-----Fração granulométrica----- } & \multicolumn{5}{|c|}{ 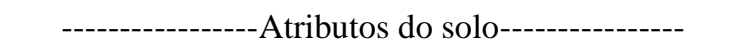 } \\
\hline \multirow{2}{*}{$\begin{array}{l}\text { Profundidade } \\
\text { (m) }\end{array}$} & Argila & Silte & Areia & \multirow[t]{2}{*}{ Classe Textural } & Ds & PT & Mac & Mic & Ucc \\
\hline & & $\%$ & & & $\mathrm{~g} \mathrm{~cm}^{-3}$ & $\mathrm{~m}^{3} \mathrm{~m}^{-3}$ & $\mathrm{~m}^{3} \mathrm{~m}^{-3}$ & $\mathrm{~m}^{3} \mathrm{~m}^{-3}$ & $\mathrm{~g} \mathrm{~g}^{-1}$ \\
\hline $0-0,10$ & 45 & 15 & 40 & Argilosa & 1,23 & 0,55 & 0,23 & 0,32 & 0,24 \\
\hline $0,10-0,20$ & 47 & 16 & 37 & Argilosa & 1,41 & 0,48 & 0,15 & 0,32 & 0,24 \\
\hline $0,20-0,40$ & 52 & 12 & 36 & Argilosa & 1,35 & 0,5 & 0,16 & 0,34 & 0,24 \\
\hline
\end{tabular}

Ds: densidade do solo; PT: porosidade total; Mac: macroporosidade; Mic: microporosidade; Ucc: umidade na capacidade de campo. Fonte: Autores.

Os dados da precipitação pluviométrica observados durante a condução do ensaio foram utilizados para cálculo do balanço hídrico, seguindo a metodologia de Rolim, Sentelhas e Barbieri (1998), apresentado na Figura 1.

Figura 1. Balanço Hídrico Mensal obtidos na Estação Meteorológica Automática - E.M.A da UFSCar - Centro de Ciências Agrárias, Araras-SP, safra 2017/18.

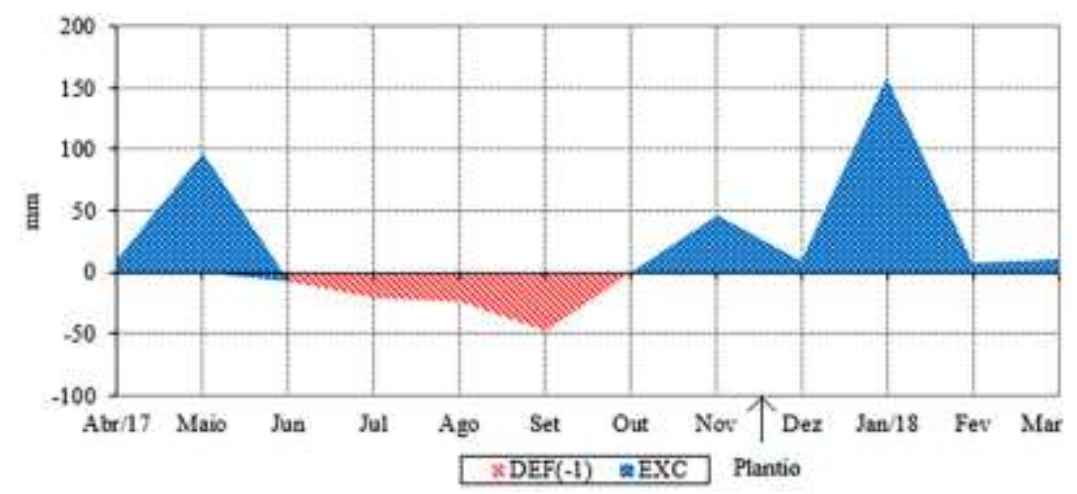

Valores de deficiência hídrica (DEF) e excedente (EXC). Indicação da data de plantio dos sistemas de cultivo. Fonte: Autores. 


\subsection{Delineamento experimental}

O delineamento experimental foi em blocos casualizados, com quatro repetições. Os tratamentos consistiram em três consórcios de milho com: Crotalaria spectabilis (MC) (5 plantas $\mathrm{m}^{-1}$ ); Cajanus cajan (MG) (5 plantas $\mathrm{m}^{-1}$ ) e Crotalaria spectabilis e Cajanus cajan (MCG) (10 plantas $\mathrm{m}^{-1}$, cinco plantas de cada adubo verde), semeados na linha do milho e três tratamentos controles: milho (M); Crotalaria spectabilis (C) e Cajanus cajan (G), solteiros. A densidade de plantas no consórcio foi baseada no Sistema Santa Brígida (Kappes; Zancanaro, 2016). A parcela experimental foi formada por 5 linhas de milho com $5 \mathrm{~m}$ de comprimento, com espaçamento entrelinhas de $0,7 \mathrm{~m}$.

\subsection{Instalação e condução do experimento}

O preparo do solo, foi realizado com uma operação de aração e uma de gradagem niveladora e, em seguida, no dia 16 de novembro de 2017 semeou-se o milho intervarietal AGRICOM 340, na densidade de quatro sementes por metro linear

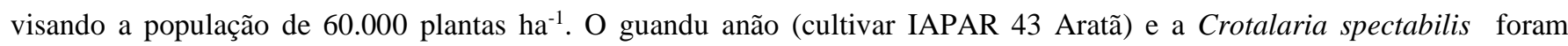
semeados simultaneamente ao milho, na mesma linha de plantio, na densidade de 25 e $15 \mathrm{Kg} \mathrm{ha}^{-1}$, respectivamente, desejando a população final de cinco plantas por metro.

Para a adubação, utilizou-se composto orgânico comercial Visafertil ${ }^{\circledR}$, distribuído sobre o solo, na linha de plantio, no estádio V2 (duas folhas expandidas) do milho. A dose aplicada foi de $13 \mathrm{t} \mathrm{ha}^{-1}$. $\mathrm{O}$ composto orgânico apresentou: $\mathrm{C}=13,1 \%$; $\mathrm{N}=1,30 \% ; \mathrm{P}_{2} \mathrm{O}_{5}=3,13 \% ; \mathrm{K}_{2} \mathrm{O}=1,62 \% ; \mathrm{CaO}=11,11 \% ; \mathrm{MgO}=0,98 \% ; \mathrm{SO}_{4}=1,50 \% ; \mathrm{Cu}=69,2 \mathrm{mg} \mathrm{kg}^{-1} ; \mathrm{Fe}=561,4 \mathrm{mg} \mathrm{kg}$

${ }^{1} ; \mathrm{Mn}=511,2 \mathrm{mg} \mathrm{kg}^{-1} ; \mathrm{Zn}=766 \mathrm{mg} \mathrm{kg}^{-1} ; 22,58 \%$ de matéria orgânica; $37,4 \%$ de umidade e $\mathrm{pH}=8,0$.

O controle das plantas espontâneas foi realizado nos estádios V5 (cinco folhas expandidas) e V8 (oito folhas expandidas) do milho, com roçadeira de fio.

\subsection{Temperatura e Umidade do solo}

A temperatura do solo foi medida com termômetro SOLOTERM MOD.1200, inserindo a haste metálica nas profundidades de $0-0,10 \mathrm{~m}, 0,10-0,20 \mathrm{~m}$ e 0,20-0,40 m, nas linhas e entrelinhas centrais de cada parcela. As leituras foram realizadas às 8:00, 12:00 e 17:00 horas, nos estádios fenológicos do milho: V5 (cinco folhas expandidas), aos 41 dias após semeadura (DAS); V8 (oito folhas expandidas), aos 60 DAS, onde a exigência hídrica do milho começa a crescer; VT (pendoamento), aos 68 DAS; R2 (grão bolha d'água), aos 82 DAS; R4 (grão pastoso), aos 96 DAS e R6 (maturidade fisiológica dos grãos), aos 127 DAS.

A umidade do solo também foi determinada nos estádios fenológicos supramencionados. Para tanto, foi coletada uma amostra de solo, para cada profundidade de 0-0,10, e 0,10-0,20 e 0,20-0,40 m, na linha central de plantio do milho de cada parcela experimental, nos horários de 8:00, 12:00 e 17:00 horas. As amostras de solo foram retiradas com trado de rosca manual e armazenadas em recipiente de alumínio, impermeável. As amostras foram vedadas e pesadas em balança semianalítica, para determinar a massa do solo úmido. Posteriormente, foram secas em estufa com circulação de ar, a $105^{\circ} \mathrm{C}$, por 24 horas e pesadas em balança semi-analítica para a determinação da massa seca do solo (Embrapa, 2017).

\subsection{Massa de matéria seca do milho}

Para a massa de matéria seca, foram coletadas três plantas de milho por parcela, nos estádios V4, V8 e VT (23, 58 e 64 DAS, respectivamente). As amostras foram levadas para a estufa com ventilação forçada de ar a $65^{\circ} \mathrm{C}$ até apresentarem massa constante e, posteriormente pesadas em balança semi-analítica. A média da massa seca por planta de milho foi multiplicada pelo número de plantas de milho por hectare e os valores apresentados em $\mathrm{kg} \mathrm{ha}^{-1}$. 


\subsection{Massa de Matéria Seca dos Adubos Verdes}

Para a determinação da massa de matéria seca dos adubos verdes nos consórcios foram coletadas a parte aérea de todas as plantas por parcela de adubos verdes presentes em um metro linear, nos estádios fenológicos V4, V8, VT e R6 do milho (23, 58, 64 e 120 DAS, respectivamente). Posteriormente, as amostras foram levadas para a estufa com ventilação forçada de ar a $65^{\circ} \mathrm{C}$ até apresentarem massa constante. Os valores de massa de matéria seca dos adubos verdes foram apresentados em kg ha-1. A massa de matéria seca dos cultivos solteiros de guandu anão e de Crotalaria spectabilis foram avaliadas nos estádios fenológicos V8 VT e R6 do milho, coletando-se a parte aérea dos adubos verdes em $1 \mathrm{~m}^{2}$, sem repetição.

\subsection{Análises estatísticas}

A variável temperatura do solo foi analisada separadamente para linha e entrelinha de plantio, em esquema fatorial 6x3x3, com quatro repetições, sendo seis sistemas de cultivo (MC, MG, MCG, M, C e G), três profundidades de solo (0-0,10; 0,10-0,20 e 0,20-0,40m) e três horários de avaliação (08:00; 12:00; 17:00 horas). Foram utilizadas as médias das temperaturas de todos os estádios fenológicos do milho.

A variável umidade do solo foi analisada em esquema fatorial 6x3x6 com quatro repetições, sendo seis sistemas de cultivo; três profundidades do solo $(0-0,10 ; 0,10-0,20$ e 0,20-0,40m) e seis estádios fenológicos do milho (V5, V8, VT, R2, R4 e R6).

Os resultados de temperatura e umidade do solo, massa de matéria seca do milho e dos adubos verdes no consórcio foram submetidos a análise de variância e as medias comparadas pelo teste de Scott-Knott, a 5\% de significância. As análises foram realizadas no software SISVAR (Versão 5.6) para Windows (Ferreira, 2000).

\section{Resultados e Discussão}

\subsection{Temperatura do solo}

Para temperatura do solo na linha e entrelinha de plantio, as análises de variância revelaram efeitos das interações horário x profundidade do solo (Tabela 2) e horários x sistemas de cultivo (Tabela 3).

Tabela 2. Amplitudes e Temperaturas do solo $\left({ }^{\circ} \mathrm{C}\right)$ em função dos horários e profundidades de avaliação. Araras-SP, safra $2017 / 18$.

\begin{tabular}{|c|c|c|c|}
\hline \multicolumn{4}{|c|}{ Temperatura do Solo $\left({ }^{\circ} \mathrm{C}\right)$} \\
\hline & & dade (m) & \\
\hline \multirow{2}{*}{ Horários } & $0-0,10$ & $0,10-0,20$ & $0,20-0,40$ \\
\hline & \multicolumn{2}{|c|}{ Linha } & \\
\hline $8: 00 \mathrm{~h}$ & $24,61 \mathrm{Aa}$ & $25,06 \mathrm{Bb}$ & $25,31 \mathrm{Bc}$ \\
\hline $12: 00 \mathrm{~h}$ & $24,39 \mathrm{Aa}$ & $24,40 \mathrm{Aa}$ & 24,49 Аa \\
\hline $17: 00 \mathrm{~h}$ & $26,85 \mathrm{Bc}$ & $26,10 \mathrm{Cb}$ & $25,68 \mathrm{Ca}$ \\
\hline Amplitude & 2,46 & 1,7 & 1,19 \\
\hline \multirow[t]{2}{*}{$\mathrm{CV}(\%)$} & 4,88 & $\mathrm{Fc}=24,585$ & $p<0,01$ \\
\hline & \multicolumn{2}{|c|}{ Entrelinha } & \\
\hline $8: 00 \mathrm{~h}$ & $24,72 \mathrm{Aa}$ & $25,09 \mathrm{Bb}$ & $25,18 \mathrm{Bb}$ \\
\hline $12: 00 \mathrm{~h}$ & $24,60 \mathrm{Aa}$ & $24,52 \mathrm{Aa}$ & $24,59 \mathrm{Aa}$ \\
\hline $17: 00 \mathrm{~h}$ & $27,35 \mathrm{Bb}$ & $26,27 \mathrm{Ca}$ & $26,10 \mathrm{Ca}$ \\
\hline Amplitude & 2,75 & 1,75 & 1,51 \\
\hline $\mathrm{CV}(\%)$ & 5,41 & $\mathrm{Fc}=38,190$ & $p<0,01$ \\
\hline
\end{tabular}

Médias seguidas pelas mesmas letras, maiúscula na coluna e minúscula na linha, não diferem entre si pelo teste de Scott-Knott, em nível de $5 \%$ de significância. Fonte: Autores. 
As 8 horas, a temperatura do solo, tanto na linha como na entrelinha de plantio, aumentou com a profundidade. As 12 horas não houve diferença entre as profundidades de solo, na linha e entrelinha de plantio. Porém, as 17 horas, na linha de plantio, a temperatura reduziu com a profundidade do solo. Já na entrelinha, a temperatura foi maior na profundidade de 0-10 $\mathrm{m}$, e manteve-se igual nas profundidades de $0,10-0,20 \mathrm{~m}$ e de $0,20-0,40 \mathrm{~m}$ do solo (Tabela 2). Tanto na linha quanto na entrelinha de plantio, a maior temperatura do solo foi verificada as 17 horas, em todas as profundidades (Tabela 2). O fluxo de calor é mais intenso nas camadas superficiais do solo, ganhando ou perdendo calor mais rapidamente em função da temperatura do ar. De acordo com Dantas et al. (2017), há correlação positiva entre a temperatura do ar e a temperatura do solo apenas na superfície e na profundidade de 0,05-0,10 m, e correlação negativa nas camadas inferiores $(0,30$ e 0,50 m). Já para as camadas mais profundas, os fenômenos de condução e convecção assumem o papel principal na transferência de calor, recebendo calor durante o dia da camada superficial. Para o solo em estudo, com perfil textural argiloso, homogêneo em profundidade, densidade do perfil entre 1,23 e $1,41 \mathrm{~g} \mathrm{~cm}^{-3}$ e umidade do solo próximas a capacidade de campo (Tabela 1), os fenômenos de condução e convecção são capazes de transferir e reter o calor em profundidade. Quanto maior o valor de densidade e umidade do solo, maior é o contato entre as partículas e o fluxo de massa, aumentando a condutividade térmica (Farouki, 1986; Kojima et al., 2018). Mas esses fenômenos são mais lentos na transferência de calor provocando um atraso no aquecimento em profundidade.

Resultados semelhantes foram verificados por Oliveira et al. (2010) e Gasparim et al. (2005), em que as 8:00 horas foram observados os maiores valores de temperatura dos solos em profundidade. Além de resultados semelhantes para o horário das 17:00, com os menores valores de temperatura do solo na camada mais profunda, devido a camada superficial acompanhar a temperatura ambiente, maior nesse horário. Os autores atribuem os resultados ao maior teor de água no solo em profundidade no período chuvoso, que favorece a transferência de calor por convecção para as camadas profundas, e para o caso em estudo, a umidade do solo é próxima a capacidade de campo de $24 \%$ em todas as profundidades analisadas.

Para o horário das 12 horas, em função das camadas analisadas, não ocorreu diferença, uma vez que a transferência de calor entre as camadas está mais equilibrada, tanto na linha como entrelinha de plantio.

Já para as camadas analisadas, nos horários estudados, 0-0,10 m apresenta a maior amplitude térmica, tanto na linha, com $2,46^{\circ} \mathrm{C}$, como na entrelinha, com $2,75^{\circ} \mathrm{C}$. As 12 horas, quando o solo está recebendo maior energia térmica pela radiação solar, a temperatura reduziu aproximadamente $0,5^{\circ} \mathrm{C}$ nas camadas $0,10-0,20$ e $0,20-0,40 \mathrm{~m}$, tanto na linha como na entrelinha de plantio, em comparação ao horário das 8:00 horas (Tabela 2). A energia térmica líquida necessária para aquecer o solo foi suficiente apenas após as 12 horas, acarretando aumentos de aproximadamente $1,5^{\circ} \mathrm{C}$ nos valores de temperatura as 17 horas, tanto na linha como entrelinha, em todas as profundidades (Tabela 2).

Analisando os efeitos dos sistemas de cultivos para a temperatura do solo dentro dos horários de avaliação, na linha de plantio, os sistemas não diferiram entre si as 8 horas (Tabela 3). Provavelmente, nesse horário, o solo recebeu pouca incidência de radiação solar, ainda não sendo possível observar diferenças bruscas. 
Tabela 3. Amplitudes e Temperatura do solo $\left({ }^{\circ} \mathrm{C}\right)$ em função dos horários de avaliação e dos sistemas de cultivo. Araras-SP, safra $2017 / 18$.

\begin{tabular}{|c|c|c|c|c|}
\hline & \multicolumn{3}{|c|}{ Temperatura do Solo $\left({ }^{\circ} \mathrm{C}\right)$} & Amplitude \\
\hline \multicolumn{5}{|c|}{ Horários } \\
\hline \multirow{2}{*}{ Sistemas } & $8: 00$ & $12: 00$ & $17: 00$ & \\
\hline & \multicolumn{3}{|c|}{ Linha } & \\
\hline MG & $25,20 \mathrm{Ab}$ & $23,88 \mathrm{Aa}$ & $25,18 \mathrm{Ab}$ & 1,32 \\
\hline MC & $24,94 \mathrm{Ab}$ & $24,27 \mathrm{Aa}$ & $26,16 \mathrm{Bc}$ & 1,89 \\
\hline MCG & $24,79 \mathrm{Ab}$ & $24,24 \mathrm{Aa}$ & $26,22 \mathrm{Bc}$ & 1,98 \\
\hline M & $24,95 \mathrm{Aa}$ & $24,32 \mathrm{Ab}$ & $26,54 \mathrm{Cc}$ & 2,22 \\
\hline $\mathrm{C}$ & $25,07 \mathrm{Aa}$ & $25,18 \mathrm{Ba}$ & $26,84 \mathrm{Cb}$ & 1,77 \\
\hline $\mathrm{G}$ & $24,97 \mathrm{Aa}$ & $24,82 \mathrm{Ba}$ & $26,79 \mathrm{Cb}$ & 1,97 \\
\hline $\mathrm{CV}(\%)$ & 4,88 & $\mathrm{Fc}=5,351$ & \multicolumn{2}{|c|}{$p<0,01$} \\
\hline \multicolumn{5}{|c|}{ Entrelinha } \\
\hline MG & $25,11 \mathrm{Bb}$ & $24,24 \mathrm{Aa}$ & $25,89 \mathrm{Ac}$ & 1,65 \\
\hline MC & $25,23 \mathrm{Bb}$ & $24,52 \mathrm{Ba}$ & $26,14 \mathrm{Ac}$ & 1,62 \\
\hline MCG & $25,04 \mathrm{Bb}$ & $24,51 \mathrm{Ba}$ & $26,67 \mathrm{Cc}$ & 2,16 \\
\hline $\mathrm{M}$ & $24,94 \mathrm{Ba}$ & $24,89 \mathrm{Ca}$ & $27,05 \mathrm{Db}$ & 2,16 \\
\hline $\mathrm{C}$ & $25,06 \mathrm{Ba}$ & $25,34 \mathrm{Da}$ & $27,26 \mathrm{Db}$ & 2,20 \\
\hline $\mathrm{G}$ & $24,59 \mathrm{Ab}$ & $24,10 \mathrm{Aa}$ & $26,43 \mathrm{Bc}$ & 2,33 \\
\hline $\mathrm{CV}(\%)$ & 5,41 & $\mathrm{Fc}=6,603$ & \multicolumn{2}{|c|}{$p<0,01$} \\
\hline
\end{tabular}

Médias seguidas pelas mesmas letras, maiúscula na coluna e minúscula na linha, não diferem entre si pelo teste de Scott-Knott, em nível de 5\% de significância. Milho-Guandu anão (MG); Milho-Crotalaria spectabilis (MC); Milho-Crotalaria spectabilis-Guandu anão (MCG); Milho solteiro (M); Crotalaria spectabilis solteira (C); guandu anão solteiro (G). Fonte: Autores.

No entanto, as 12 horas, as maiores temperaturas do solo foram observadas nos sistemas C e G, e não houve diferença entre os demais sistemas de cultivo. Também, as 17 horas as maiores temperaturas foram verificadas nos sistemas C, G e M, e a menor temperatura no consórcio MG (Tabela 3). Os sistemas solteiros de cultivo possuem uma produção de matéria seca menor quando comparados aos sistemas consorciados (Tabela 4; Figura 2), deixando os solos mais expostos a incidência da radiação solar direta, o que contribuiu, de forma geral, para as maiores amplitudes térmicas dentre os sistemas de cultivo (Tabela 3).

Observando a posição da entrelinha de plantio as 8 horas, a menor temperatura registrada foi no G (Tabela 3), sem ocorrer diferenças nos demais sistemas. Já as 12 horas, a maior temperatura foi verificada no sistema $\mathrm{C}$ e as 17 horas nos sistemas C e M, e as menores no MG e MC (Tabela 3). Novamente, observa-se que os consórcios, em geral, proporcionaram redução das temperaturas do solo, devido a maior massa de matéria seca produzida nesses sistemas (Tabela 4; Figura 2), contribuindo para maior cobertura do solo. Gitti et al. (2012), estudando as épocas de semeadura da crotalária em consórcio com milho, semeadas na entrelinha, encontrou maior matéria seca nos consórcios de milho com Crotalaria spectabilis e Crotalaria juncea. 
Tabela 4. Acúmulo de massa de matéria seca $\left(\mathrm{kg} \mathrm{ha}^{-1}\right)$ dos adubos verdes e do milho nos diferentes sistemas de cultivo e estádios fenológicos. Araras-SP, safra 2017/18.

\begin{tabular}{ccccc}
\hline \multicolumn{5}{c}{ Matéria Seca Adubos Verdes $\left(\mathrm{kg} \mathrm{ha}^{-1}\right)$} \\
\hline Sistemas & V4 & V8 & VT & R6 \\
\cline { 2 - 5 } MG & $14,10 \mathrm{Ac}$ & $238,54 \mathrm{Ac}$ & $773,91 \mathrm{Ab}$ & $3812,36 \mathrm{Aa}$ \\
MC & $11,35 \mathrm{Ab}$ & $223,86 \mathrm{Ab}$ & $499,84 \mathrm{Ab}$ & $1949,06 \mathrm{Ba}$ \\
MCG & $9,28 \mathrm{Ab}$ & $341,55 \mathrm{Ab}$ & $644,64 \mathrm{Ab}$ & $2328,65 \mathrm{Ba}$ \\
\hline $\mathrm{CV}(\%)$ & 39,76 & $\mathrm{Fc}=7,086$ & $p<0,01$ \\
\hline MG & $147,2 \mathrm{Ab}$ & $3639,75 \mathrm{Ca}$ & $3756,15 \mathrm{Da}$ & \\
\hline MC & $274,0 \mathrm{Ab}$ & $8120,50 \mathrm{Aa}$ & $8270,55 \mathrm{Aa}$ & -- \\
MCG & $239,5 \mathrm{Ab}$ & $5393,60 \mathrm{Ba}$ & $5558,85 \mathrm{Ca}$ & -- \\
M & $230,5 \mathrm{Ab}$ & $5526,28 \mathrm{Ba}$ & $6356,40 \mathrm{Ba}$ & - \\
\hline $\mathrm{CV}(\%)$ & 27,75 & $\mathrm{Fc}=3,681$ & $p<0,01$ & \\
\hline
\end{tabular}

Médias seguidas pelas mesmas letras, maiúscula na coluna e minúscula na linha, não diferem entre si pelo teste de Scott-Knott, em nível de 5\% de significância. Milho-Guandu anão (MG); Milho-Crotalaria spectabilis (MC); Milho-Crotalaria spectabilis-Guandu anão (MCG); Milho solteiro (M). Fonte: Autores.

Analisando a variação da temperatura do solo ao longo dos horários dentro de cada sistema, tanto na linha como na entrelinha de plantio, as maiores temperaturas do solo são observadas as 17 horas, exceto para o sistema MG na linha de plantio, onde a temperatura do solo as 17 horas é semelhante a observada as 8 horas, o qual, apresenta a menor amplitude térmica diária dentre os sistemas (Tabela 3). Provavelmente, devido a maior produção de massa de matéria seca do guandu anão nesse sistema (Tabela 4) em comparação aos adubos verdes solteiros (Figura 2).

Figura 2. Acúmulo de matéria seca da Crotalaria spectabilis e Cajanus cajan nos sistemas solteiros aos 58,64 e 120 dias após a semeadura (DAS). Araras-SP, safra 2017/18.

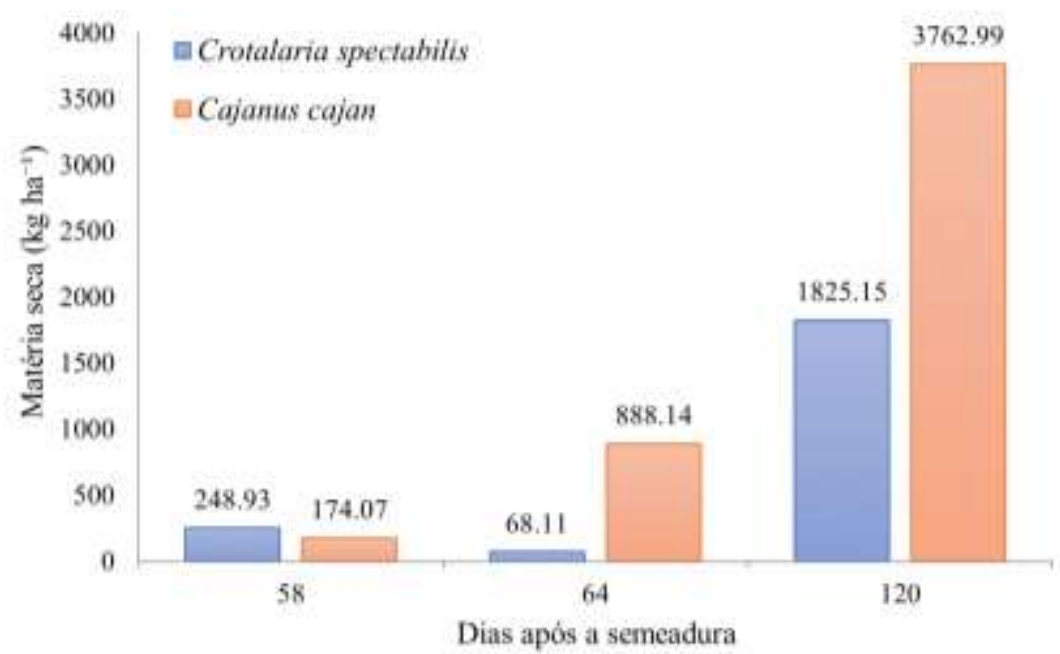

Fonte: Autores.

Em condições de elevada pluviosidade, o guandu anão apresenta desenvolvimento vegetativo vigoroso, protegendo o solo pelo acúmulo de matéria verde (Alvarenga et al., 1995). Mesmo, sendo utilizada a variedade anã, quando o guandu é semeado em novembro e ocorre alta precipitação pluviométrica, como verificado na Figura 1, o mesmo apresenta elevado crescimento, neste caso, de 3812,36 kg ha-1 no consórcio MG (Tabela 4), ultrapassando a produção de matéria seca dos 
cultivares normais, que de acordo com Castro e Devide (2015) alcançam $1390 \mathrm{~kg} \mathrm{ha}^{-1}$ de matéria seca, quando na densidade de 20 sementes por metro em consórcio com milho.

\subsection{Umidade do solo}

A análise de variância para umidade do solo revelou efeito das interações para sistemas de cultivo x profundidade do solo (Tabela 5) e sistemas de cultivo x estádios fenológicos do milho (Tabela 6). Nas profundidades de 0-0,10 e 0,10-0,20 m, o sistema MG apresentou maiores valores de umidade do solo (Tabela 5).

Tabela 5. Umidade do solo (\%) em função dos sistemas de cultivo e profundidades do solo, Araras, SP, safra $2017 / 18$.

\begin{tabular}{cccc}
\hline \multicolumn{3}{c}{ Umidade do Solo (\%) } \\
\hline Sistemas & \multicolumn{3}{c}{ Profundidade $(\mathrm{cm})$} \\
MG & $0-0,10$ & $0,10-0,20$ & $0,20-0,40$ \\
MC & $26,67 \mathrm{Dc}$ & $22,97 \mathrm{Bb}$ & $21,19 \mathrm{Aa}$ \\
MCG & $25,12 \mathrm{Cc}$ & $21,76 \mathrm{Aa}$ & $22,58 \mathrm{Bb}$ \\
M & $23,24 \mathrm{Bb}$ & $22,09 \mathrm{Aa}$ & $22,92 \mathrm{Cb}$ \\
$\mathrm{C}$ & $25,03 \mathrm{Cc}$ & $22,19 \mathrm{Aa}$ & $23,41 \mathrm{Cb}$ \\
$\mathrm{G}$ & $21,84 \mathrm{Aa}$ & $21,54 \mathrm{Aa}$ & $22,17 \mathrm{Ba}$ \\
$\mathrm{CV}(\%)$ & $21,72 \mathrm{Aa}$ & $21,76 \mathrm{Aa}$ & $23,00 \mathrm{Cb}$ \\
\hline
\end{tabular}

Médias com letras iguais, maiúscula na coluna e minúscula na linha, não diferem entre si pelo teste de Scott-Knott, em nível de 5\% de significância. Consórcio de milho-Guandu anão (MG); consórcio milho-Crotalaria spectabilis (MC); consórcio milho-Crotalaria spectabilis-Guandu anão (MCG); milho solteiro (M); Crotalaria spectabilis solteira $(\mathrm{C})$; guandu anão solteiro $(\mathrm{G})$.

Fonte: Autores.

Já na profundidade de 0,20-0,40 m, o MG apresentou o menor valor de umidade do solo (Tabela 5), provavelmente, devido ao estabelecimento da competição por água entre o milho e a fabácea. Os resultados podem indicar que a competição se estabelece em profundidades do solo superiores a $0,20 \mathrm{~m}$. O guandu possui sistema radicular vigoroso e profundo, destaca-se a raiz principal (pivotante), que pode alcançar mais de $2 \mathrm{~m}$ de profundidade (Godoy et al., 2009; Alvarenga et al., 1996). Dela originam-se várias outras, mais finas, secundárias, localizadas principalmente nos 0,30 m de profundidade do solo (Souza et al., 2007). Também é nessa profundidade $(0,30 \mathrm{~m})$ que se concentram a maior parte das raízes do milho (Bassoi, et al., 1994). Apesar de não ter ocorrido déficit hídrico durante a condução do experimento (Figura1), a competição entre o milho e o guandu anão no consórcio é provável, quando se observa a redução da massa de matéria seca do milho no consórcio com o guandu em comparação com os demais consórcios e ao milho solteiro (Tabela 4).

Para os sistemas M, MCG e G, a umidade do solo foi superior na profundidade 0,20-0,40 m. Portanto, ao inserir a crotalária no consórcio com o M+G, reduz-se a competição por água na profundidade de 0,20-0,40 m. O sistema radicular das crotalárias concentram suas raízes na camada de 0-0,10 m de profundidade (Reinert et al., 2008). Assim, provavelmente estabeleceu-se uma competição entre a C. spectabilis com o guandu anão, reduzindo o desenvolvimento do último. Tal fato, corrobora com os resultados observados na Figura 2, em que o acúmulo de matéria seca do milho no consórcio crotalária + guandu anão é superior ao do milho em consórcio com o guandu anão, e superior ao milho solteiro até o estádio V8.

Analisando o desdobramento da interação estádios fenológicos do milho x sistemas de cultivo (Tabela 6), no estádio V5 a umidade do solo foi semelhante em todos os sistemas de cultivo (Tabela 6). 
Tabela 6. Umidade do solo (\%) ao longo dos estádios fenológicos do milho nos diferentes sistemas de cultivo. Araras-SP, safra $2017 / 18$.

\begin{tabular}{|c|c|c|c|c|c|c|}
\hline \multicolumn{7}{|c|}{ Umidade do Solo (\%) } \\
\hline \multicolumn{7}{|c|}{ Estádio } \\
\hline Sistemas & V5 & V8 & $\mathrm{VT}$ & $\mathrm{R} 2$ & $\mathrm{R} 4$ & R6 \\
\hline MG & 23,25 Ac & $24,48 \mathrm{Ad}$ & $21,27 \mathrm{Bb}$ & $20,00 \mathrm{Ba}$ & $26,51 \mathrm{Be}$ & $26,00 \mathrm{Ce}$ \\
\hline $\mathrm{MC}$ & $23,27 \mathrm{Ac}$ & $26.24 \mathrm{Be}$ & $21,76 \mathrm{Bb}$ & $19,01 \mathrm{Aa}$ & $24,91 \mathrm{Ad}$ & $23,73 \mathrm{Ac}$ \\
\hline MCG & $22,93 \mathrm{Ac}$ & $24,50 \mathrm{Ad}$ & $19,21 \mathrm{Aa}$ & $20,35 \mathrm{Bb}$ & $24,76 \mathrm{Ad}$ & $24,76 \mathrm{Bd}$ \\
\hline M & $23,73 \mathrm{Ab}$ & $25,84 \mathrm{Bc}$ & $23,40 \mathrm{Cb}$ & $19,43 \mathrm{Aa}$ & $25,58 \mathrm{Ac}$ & $23,38 \mathrm{Ab}$ \\
\hline $\mathrm{C}$ & $22,23 \mathrm{Ab}$ & $24,30 \mathrm{Ac}$ & 19,18 Аа & $18,56 \mathrm{Aa}$ & $24,99 \mathrm{Ac}$ & --- \\
\hline $\mathrm{G}$ & $22,62 \mathrm{Ab}$ & $23,63 \mathrm{Ab}$ & $19,66 \mathrm{Aa}$ & $19,53 \mathrm{Aa}$ & $25,35 \mathrm{Ac}$ & --- \\
\hline $\mathrm{CV}(\%)$ & 10,02 & & $\mathrm{Fc}=3,965$ & & $p<0,01$ & \\
\hline
\end{tabular}

Médias seguidas pelas mesmas letras, maiúscula na coluna e minúscula na linha, não diferem entre si de acordo com o teste de Scott-Knott, em nível de 5\% de significância. V5 (Cinco folhas expandidas), V8 (Oito folhas expandidas), VT (Pendoamento), R2 (Bolha d'água), R4 (Pastoso) e R6 (Maturidade fisiológica do grão). Consórcio de milho-guandu anão (MG); consórcio milho-Crotalaria spectabilis (MC); consórcio milho-Crotalaria spectabilis-guandu anão (MCG); milho solteiro (M); Crotalaria spectabilis solteira (C); guandu anão solteiro (G). Fonte: autores.

Sendo as mesmas condições para todos os sistemas, e tendo como base o valor da umidade do solo na capacidade de campo de $24 \%$ (Tabela 1), o desejado era que a umidade do solo fosse mantida ou aumentada, em comparação ao estádio fenológico anterior, sendo preferido um sistema consorciado que trará os benefícios desse modelo.

Do estádio V5 ao V8, todos os sistemas apresentaram incremento na umidade do solo, com maior aumento em MC, seguido pelo M. Destaca-se que nesse período, houve o maior excedente hídrico do solo (Figura 1), justificando o aumento de umidade em todos os sistemas. No entanto, o maior aumento de umidade no estádio V8 para o MC e M, deve-se provavelmente a menor competição por água nesses sistemas.

Do V8 ao VT, a umidade do solo diminui em todos os sistemas. As menores umidades do solo foram observadas nos sistemas MCG, C e G, e a maior umidade no M (Tabela 6). De acordo com Allen et al. (1998), a duração do ciclo fenológico do milho grão, varia entre 120 e 180 dias após emergência e, a exigência hídrica é de $17 \%$ na fase 1; 28\% na fase 2; 33\% na fase 3 e $22 \%$ na fase 4 do ciclo total. Assim, entende-se que a maior exigência hídrica do milho ocorre entre os estádios V8 e VT, justamente onde verificou-se a maior umidade do solo no M, o que indica uma possível competição por água entre a poácea e os adubos verdes nesse período.

Já do estádio VT ao R2, os sistemas consorciados, exceto o MCG, e o M apresentam diminuição na umidade do solo, enquanto o C e G mantiveram a umidade (Tabela 6). No R2 as maiores umidades foram observadas em MCG e MG (Tabela 6). Nesse período a exigência hídrica do milho ainda é alta (Wagner et al., 2013), e a maior umidade no solo nos sistemas MCG e MG é benéfica.

Observando o intervalo dos estádios fenológicos R2 a R6 a exigência hídrica do milho é mínima, pois aproxima-se a colheita dos grãos e, nesses as maiores umidades do solo foram verificadas no MG em R4, e, para o estádio R6, no MG seguido pelo MCG (Tabela 6). Esses sistemas foram os que apresentaram a maior produção de massa de matéria seca (Figura 2) e, provavelmente em função da maior cobertura do solo, houve menor evaporação. Acrescenta-se também que aos 120 DAS a Crotalaria spectabilis e o guandu anão já completaram o ciclo e encontram-se em senescência (Braz et al., 2015; Teodoro et al., 2011).

\section{Conclusão}

As maiores temperaturas do solo ocorrem nos horários das 17 horas, independentemente da posição ou da profundidade. Os sistemas consorciados apresentam menores temperaturas quando comparados com os sistemas solteiros. Ocorre competição por água em profundidades do solo superiores a 0,20 m entre o milho e o guandu anão, entre os estádios 
fenológicos V8 ao R2 do milho. Inserindo a Crotalaria spectabilis no consórcio de milho + guandu anão, reduz-se a competição por água na profundidade de 0,20-0,40 m.

\section{Agradecimentos}

Este estudo foi financiado pela Coordenação de Aperfeiçoamento de Pessoal de Nível Superior (CAPES), Brasil, Finance Code 001.

\section{Referências}

Allen, R. G., Pereira, L. S., Raes, D., \& Smith, M. (1998). Crop evapotranspiration: guidelines for computing crop water requirements. FAO Irrigation and Drainage Paper 56.

Alvarenga, R. C., Cobucci, T., Kluthcouski, J., Wruck, F. J., Cruz, J. C., \& Gontijo Neto, M. M. (2006). A cultura do milho na integração lavoura-pecuária. Embrapa Milho e Sorgo. Circular Técnica 80.

Alvarenga, R. C., Costa, L. M., Moura Filho, W., \& Regazzi, A. J. (1995). Características de alguns adubos verdes de interesse para a conservação e recuperação de solos. Pesquisa Agropecuária Brasileira, 30 (2), 175-185.

Alvarenga, R. C., Costa, L. M., Moura Filho, W., \& Regazzi, A. J. (1996). Crescimento de raízes de leguminosas em camadas de solo compactadas artificialmente. Revista Brasileira de Ciência do Solo, 20, 313-318.

Bassoi, L. H., Fante Júnior, L., Jorge, L. A. C., Crestana, S., \& Reichardt, K. (1994). Distribuição do sistema radicular do milho em terra roxa estruturada latossólica: II. Comparação entre cultura irrigada e fertirrigada. Scientia Agricola, 51 (3), 541-548.

Brasil. (2003). Lei no 10.831, de 23 de dezembro de 2003. Dispõe sobre a agricultura orgânica e dá outras providências. Diário Oficial da República Federativa do Brasil, Seção 1, p. 8.

Braz, G. B. P., Oliveira Júnior, R. S., Constantin, J., Takano, H. K., Chase, C. A., Fornazza, F. G. F., \& Raimond, R. T. (2015). Selection of herbicides targeting the use in crop systems cultivated with showy crotalaria. Planta Daninha, 33 (3), 521-534.

Büchi, L., Wendling, M., Amossé, C., Necpalova, M., \& Charles, R. (2018). Importance of cover crops in alleviating negative effects of reduced soil tillage and promoting soil fertility in a winter wheat cropping system. Agriculture, Ecosystems and Environment, 256, 92-104.

Castro, C. M., \& Devide, A. C. P. (2015). Cultivo Orgânico de milho verde consorciado com leguminosas. Pesquisa \& Tecnologia, 12 (1), 1-5.

Chioderoli, C. A., Mello, L. M. M., Holanda, H. V., Furlani, C. E. A., Grigolli, P. J., Silva, J. O. R., \& Cesarin, A. L. (2015). Consórcio de Urochloas com milho em sistema plantio direto. Ciência Rural, (10), 1804-1810.

Conant, R. T., Ryan, M. G., Ågren, G. I., Birge, H. E., Davidson, E. A., Eliasson, P. E., Evans, S. E., Frey, S. D., Giardina, C. P., Hopkins, F. M., Hyvönen, R., Kirschbaum, M. U. F., Lavallee, J. M., Leifeld, J., Parton, W. J., Steinweg, J. M., Wallenstein, M. D., Wetterstedt., \& Bradford, M. A. (2011). Temperature and soil organic matter decomposition rates- synthesis of current knowledge and a way forward. Global Change Biology, 17 (11), $3392-3404$.

Dantas, D., Barbosa, G. P., Cunha, E. G. S., Souza, M. J. H., \& Souza, C. M. P. (2017). Temperatura do ar e do solo em diferentes profundidades, em Diamantina-MG. Caderno de Ciências Agrárias, 9 (3), 61-66.

Empresa Brasileira de Pesquisa Agropecuária. (2017). Manual de Métodos de Análise de Solo. (3ª edição) revista e ampliada Embrapa. Embrapa.

Ferreira, D. F. (2000). Análises Estatísticas por Meio do SISVAR para Windows Versão 4.0. In: 45ª Reunião Anual da Região Brasileira da Sociedade Internacional de Biometria. UFSCar, São Carlos.

Farouki, O. T. (1986). Thermal Properties of Soils. Series on Rock and Soil Mechanics. Clausthal-Zellerfeld, Germany:Trans Tech.

Gallo, A. de S., Fontanetti, A., Guimarães, N. F., Morinigo, K. P. G., \& Souza, M. D. B. (2017). Macronutrient content and accumulations in different arrangements of dwarf pigeon pea intercropped with corn. African Journal of Agricultural Research, 12 (11), 897-904.

Abdollahi, L., Munkholm, L. J., \& Garbout, A. (2014). Tillage System and Cover Crop Effects on Soil Quality: II. Pore Characteristics. Soil Science Society of America Journal, 78 (1), 271-279

Gasparim, E., Ricieri, R. P., Silva, S. L., Dallacort, R., \& Gnoatto, E. (2005). Temperatura no perfil do solo utilizando duas densidades de cobertura e solo nu. Acta Scientiarum. Agronomy, 27 (1), 107-115.

Gitti, D. C., Arf, O., Portugal, J. R., Corsini, D. C. D. C., Rodrigues, R. A. F., \& Kaneko, F. H. (2012). Coberturas vegetais, doses de nitrogênio e inoculação de sementes com Azospirillum brasilense em arroz de terras altas no sistema plantio direto. Bragantia, 71 (4) $509-517$.

Godoy, R., Bacchi, O. O. S., Moreira, F. A., \& Reichardt, K. (2009). Evaluation of pigeon pea lines for biological soil decompaction. International Journal of Agronomy, 2009, 1-7.

Heinrichs, R., Vitti, G. C., Moreira, A., Figueiredo, P. A. M., Fancelli, A. L., \& Corazza, E. J. (2005). Características químicas de solo e rendimento de fitomassa de adubos verdes e de grãos de milho, decorrente do cultivo consorciado. Revista Brasileira de Ciência do Solo, 29 (1), $71-79$. 
Research, Society and Development, v. 10, n. 14, e539101422443, 2021

(CC BY 4.0) | ISSN 2525-3409 | DOI: http://dx.doi.org/10.33448/rsd-v10i14.22443

Heinze, J., Gensch, S., Weber, E., \& Joshi, J. (2017). Soil temperature modifies effects of soil biota on plant growth. Journal of Plant Ecology, 10 (5), 808821.

Kappes, C., \& Zancanaro, L. Sistemas de consórcios de braquiária e de crotalárias com a cultura do milho. Revista Brasileira de Milho e Sorgo, 14 (2), 219 234.

Kaspart, C., \& Bland, W. L. (1992). Soil temperature and root growth. Soil Science, 154 (4), 290-299.

Kojima, Y., Heitman, J. L., Sakai, M., Kato, C., \& Horton, R. (2018). Bulk density effects on soil hydrologic and thermal characteristics: A numerical investigation. Hydrological Processes, 32 (14), 2203-2216.

Lal, R. (1974). Soil temperature, soil moisture and maize yield from mulched and unmulched tropical soils. Plant and Soil, 40, 129-143.

Nunes, M. R., Van Es, H. M., Schindelbeck, R., Ristow, A. J., \& Ryan, M. (2018). No-till and cropping system diversification improve soil health and crop yield. Geoderma, 328, 30-43.

Oliveira, M. L., Ruiz, H. A., Costa, L. M., \& Schaefer, C. E. G. R. (2005). Flutuações de temperatura e umidade do solo em resposta à cobertura vegetal. Revista Brasileira de Engenharia Agrícola e Ambiental, 9 (4), 535-539.

Oliveira, S. S., Fideles Filho, J., Oliveira, S. V., \& Araújo, T. S. (2010). Difusividade térmica do solo de campina grande para dois períodos do ano. Revista de Geografia, 27 (2), 179-189.

Pacheco, L. P., Pires, F. R., Monteiro, F. P., Procópio, S. O., Assis, R. L., Carmo, M. L., \& Petter, F. A. (2008). Desempenho de plantas de cobertura em sobressemeadura na cultura da soja. Pesquisa Agropecuária Brasileira, 43 (7), 815-823.

Reinert, D. J., Albuquerque, J. A., Reichert, J. M., Aita, C., \& Andrada, M. M. C. (2008). Limites críticos de densidade do solo para o crescimento de raízes de plantas de cobertura em Argissolo vermelho. Revista Brasileira de Ciência do Solo, 32 (5), 1805-1816.

Rolim, G. S., Sentelhas, P. C., \& Barbieri, V. (1998). Planilhas no ambiente EXCEL TM para os cálculos de balanços hídricos: normal, sequencial, de cultura e de produtividade real e potencial. Revista Brasileira de Agrometeorologia, 6 (1), 133-137.

Santos, G. G., Silveira, V. M., Marchão, R. L., Becquer, T., \& Balbino, L. C. (2008). Macrofauna edáfica associada a plantas de cobertura em plantio direto em um Latossolo Vermelho do Cerrado. Pesquisa Agropecuária Brasileira, 43 (1), 115-122.

Snapp, S., \& Surapur, S. (2018). Rye cover crop retains nitrogen and doesn't reduce corn yields. Soil and Tillage Research, 180, 107-115.

Souza, F. H. D., Frigeri, T., Moreira, A., \& Godoy, R. (2007). Produção de sementes de guandu. Embrapa Pecuária Sudeste.

Teodoro, R. B., Oliveira, F. L., Silva, D. M. N., Fávero, C., \& Quaresma, M. A. L. (2011). Aspectos agronômicos de leguminosas para adubação verde no cerrado do alto vale do Jequitinhonha. Revista Brasileira de Ciência do Solo, 35 (2), 635-643.

Wagner, M. V., Jadoski, S. O., Maggi, M. F., Saito, L. R., \& Lima, A. S. (2013). Estimativa da produtividade do milho em função da disponibilidade hídrica em Guarapuava, PR, Brasil. Revista Brasileira de Engenharia Agrícola e Ambiental, 17 (2), 170-179.

Yoshida, F. A., \& Stolf, R. Mapeamento digital de atributos e classes de solos da UFSCar - Araras/SP. Ciência, Tecnologia e Ambiente, 3 (1), 1-11.

Zhou, Z., Jiang, L., Du, E., Hu, H., Li, Y., Chen, D., \& Fang, J. (2013). Temperature and substrate availability regulate soil respiration in tropical mountain rainforests, Hainan Island. Journal of Plant Ecology, 6 (5), 325-334. 